\title{
Die Flourishing Scale (FS) von Diener et al. liegt jetzt in einer autorisierten deutschen Fassung (FS-D) vor: Einsatz bei einer Mind-Body-medizinischen Fragestellung
}

\author{
Tobias Escha,b,c Gerald Jose ${ }^{\mathrm{d}} \quad$ Christine Gimpel $^{\mathrm{a}, \mathrm{e}} \quad$ Christel von Scheidt ${ }^{\mathrm{e}} \quad$ Andreas Michalsen $^{\mathrm{e}, \mathrm{f}}$ \\ ${ }^{\text {a }}$ Fachbereich Integrative Gesundheitsförderung, Hochschule Coburg, Deutschland \\ ${ }^{\mathrm{b}}$ Neuroscience Research Institute, State University of New York, \\ ${ }^{c}$ Division of General Medicine and Primary Care, Beth Israel Deaconess Medical Center, Harvard Medical School, Boston, USA \\ ${ }^{\mathrm{d}}$ Fachbereich Soziale Arbeit und Gesundheit, Hochschule Coburg, \\ ${ }^{\mathrm{e}}$ Immanuel-Krankenhaus Berlin, Abteilung für Naturheilkunde, \\ ${ }^{f}$ Institut für Sozialmedizin, Epidemiologie und Gesundheitsökonomie, Charité-Universitätsmedizin, Berlin, Deutschland
}

\section{Schlüsselwörter}

Flourishing $\cdot$ FS $\cdot$ FS-D $\cdot$ Mind-Body-Medizin .

Achtsamkeit · Kohärenz · Wohlbefinden · Lebensqualität · Validierung · Positive Psychologie

\section{Zusammenfassung}

Hintergrund: Die Flourishing Scale (FS) von Ed Diener et al. wurde im Rahmen einer Interventionsstudie ins Deutsche übersetzt und erstmals im deutschsprachigen Raum getestet und validiert. Methoden: Die FS ist ein kurzes 8-Item-Messinstrument. Die Items wurden aus verschiedenen Bereichen, die ein subjektives psychologisches Wohlbefinden beschreiben, zusammengesetzt. Ergebnisse: Die deutsche Version der Flourishing Scale (FS-D) erwies sich als stabiles, reliables und valides Messinstrument für das psychologische "Aufblühen" (Flourishing) bei Patienten einer Mind-Body-medizinischen Tagesklinik. Wesentliche Parameter des OriginalFragebogens konnten verifiziert werden. Schlussfolgerung: Zusätzlich werden Zusammenhänge zwischen Flourishing, Achtsamkeit, Kohärenzgefühl und gesundheitsbezogener Lebensqualität (sowie Mind-Body-Medizin) dargestellt.

\section{Einführung: Forschungsrahmen und Forschungsfrage}

Die Flourishing Scale (FS) von Ed Diener et al. [1] ist ein Messinstrument zur Erfassung des soziopsychologischen Wohlbefindens im Sinne des Aufblühens (Flourishing) der

\section{Keywords}

Flourishing $\cdot$ FS $\cdot$ FS-D $\cdot$ Mind-body medicine .

Mindfulness · Coherence - Well-being · Quality of life ·

Validation · Positive Psychology

\section{Summary}

The Flourishing Scale (FS) by Diener et al. is Now Available in an Authorized German Version (FS-D): Application in Mind-Body Medicine

Background: The Flourishing Scale (FS) of Ed Diener et al. was translated into German, tested, and validated for the first time in a German-speaking population, as part of an interventional study. Methods: The FS is a short 8-item questionnaire. Items were constructed from various areas that describe subjective psychological wellbeing. Results: The German version of FS (FS-D) proved to be a stable, reliable, and valid measuring tool for psychological flourishing in patients of a mind-body medical outpatient clinic. Basic parameters of the original scale could be verified. Conclusion: Additionally, relations between flourishing, mindfulness, sense of coherence, and health-related quality of life (as well as mindbody medicine) are demonstrated.

Persönlichkeit. Hierunter wird, kurz gefasst, die Selbsteinschätzung persönlicher perspektivischer Potenziale und Ressourcen verstanden. Die Skala und das dahinterstehende Konstrukt basieren auf zum Teil knapp 20 Jahre alten Vorarbeiten zum subjektiven Wohlbefinden (SWB) und zur geistig-

\section{KARGER}

Fax +497614520714

Information@Karger.com

www.karger.com

\section{(C) 2013 S. Karger GmbH, Freiburg}

$1661-4119 / 13 / 0204-0267 \$ 38.00 / 0$

Accessible online at:

www.karger.com/fok
Tobias Esch, M.D

Division of General Medicine and Primary Care

Beth Israel Deaconess Medical Center

Rabb 117, 330 Brookline Avenue, Boston, MA 02215, U.S.A.

tesch@bidmc.harvard.edu 


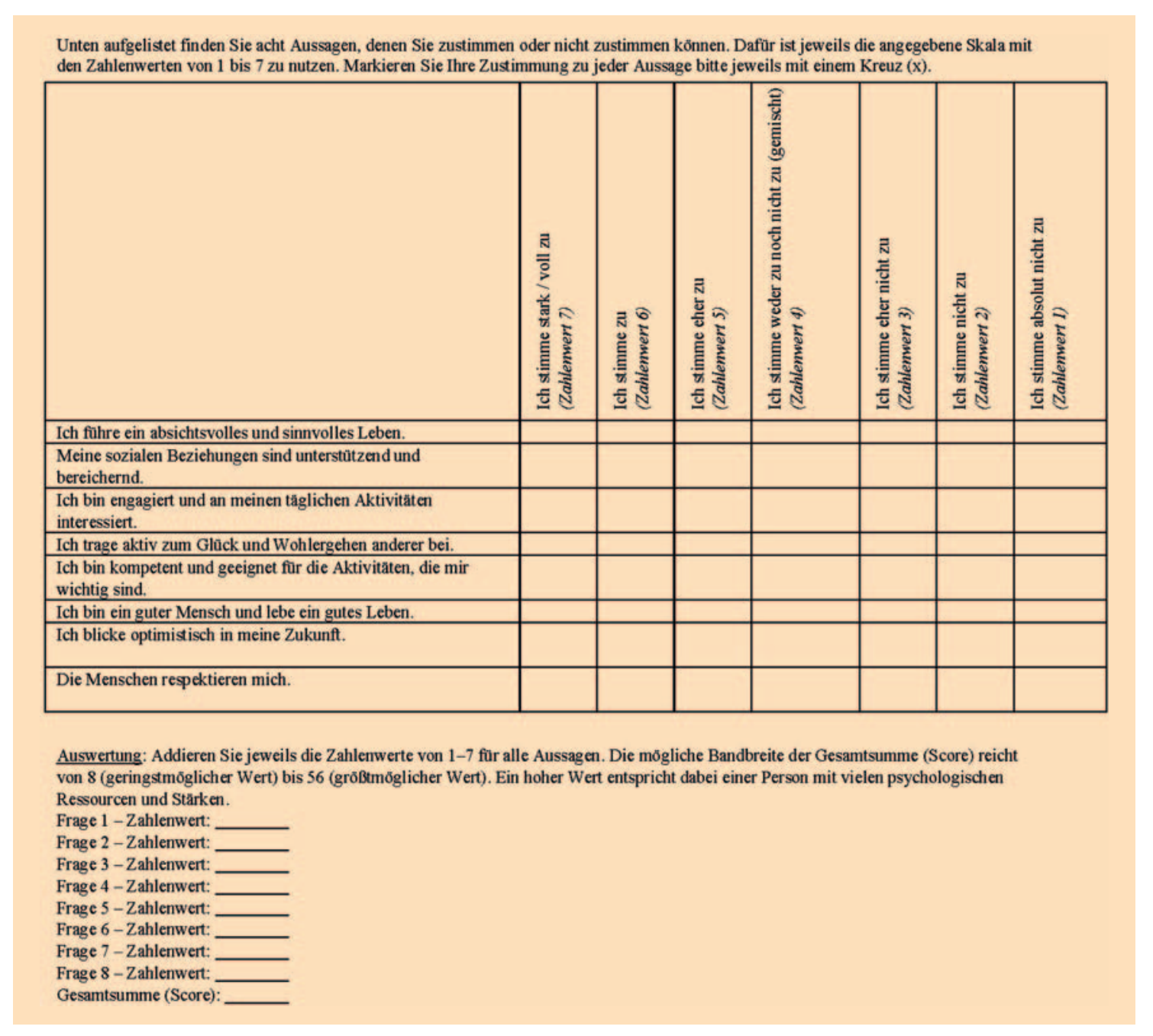

Abb. 1. Autorisierte deutsche Übersetzung (FS-D von T.E.) der FS von Ed Die-

ner et al. [1].

Unten aufgelistet finden Sie acht Aussagen, denen Sie zustimmen oder nicht zustimmen können. Dafür ist jeweils die angegebene Skala mit den Zahlenwerten von 1 bis 7 zu nutzen. Markieren Sie Ihre Zustimmung zu jeder Aussage bitte jeweils mit einem Kreuz (x).

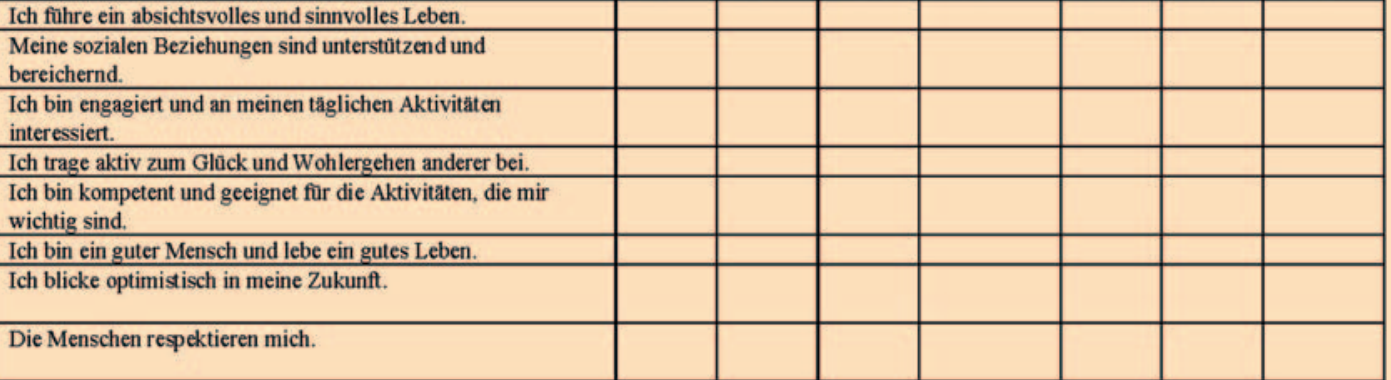

Auswertung: Addieren Sie jeweils die Zahlenwerte von 1-7 fur alle Aussagen. Die mogliche Bandbreite der Gesamtsumme (Score) reicht Ressourcen und Stărken.

Frage 1 -Zahlenwer

Frage 2 - Zahlenwer

- Zahlenwert

mentalen Gesundheit mit ihrem Bezug zu positiven Gefühlen, einem Zustand der «innerlichen Erfüllung» und der positiven subjektiven Lebenseinschätzung («Bilanz» und Projektion) [1-6]. In Ergänzung dazu haben andere Autoren beispielsweise den Funktionalitätsaspekt von Flourishing stärker betont (d.h. das Entwickeln und «optimale Leben» der eigenen Kompetenzen [7]) oder die grundlegende Fähigkeit zu einer bewussten Lebensführung $[8,9]$.

Der FS-Fragebogen stand bisher nicht in einer deutschen Version zur Verfügung. Im Rahmen einer Interventionsstudie wurde die FS ins Deutsche (FS-D) übersetzt und erstmalig 2011 im deutschsprachigen Raum getestet sowie validiert (Abb. 1).

Die Grundlagen für die Entwicklung der FS waren verschiedene Theorien zum Wohlbefinden, darunter humanistische und psychologische Ansätze, Wohlbefinden als soziales Kapital, die Flow-Theorie, aber auch Annahmen zu Glück, Optimismus und Engagement, zu Kohärenz und Sinnhaftigkeit sowie zur Altruismus-Hypothese [1, 8, 10, 11]. Folglich umfassen die 8 FS-Items Aspekte zu sozialen Beziehungen, der Bedeutung und Erfüllung des Lebens, zum Engagement und Interesse an Aktivitäten, Optimismus, Selbstrespekt so- wie zur Selbstwahrnehmung in Bezug auf Kompetenzen und Fähigkeiten.

Flourishing ist ein komplexes Konstrukt. In verschiedenen Studien wird Flourishing als eine Art des Wohlbefindens beschrieben $[1,7,12]$. Es umfasst «positive» menschliche Fähigkeiten und Kompetenzen, unter anderem Güte, psychische Kreativität, Wachstum und Resilienz [7, 12]. Für das Flourishing können unter anderem auch Meditation und eine achtsame Einstellung bedeutsam sein [13-15]. Zahlreiche Studien haben gezeigt, dass verschiedene Entspannungs- und Achtsamkeitstechniken (oder achtsamkeitsbasierte Meditation) in Verbindung mit einer Steigerung des Wohlbefindens sowie der Reduktion von Schmerz, Angst, Depression, Stress und Symptomen bei Krebserkrankungen stehen [16-23]. Achtsamkeit kann hier als eine Geisteshaltung verstanden werden, in der man den gegenwärtigen Moment mit einer nicht bewertenden, akzeptierenden, offenen und freundlichen Haltung wahrnimmt $[22,24,25]$. Achtsames Bewusstsein beinhaltet demnach mentale, psychische und physische Prozesse [26]. Für dessen wachsende Bedeutung im medizinisch-psychologischen Kontext ist hervorzuheben, dass Achtsamkeit heute als ein trainierbarer Bewusstseinszustand angesehen wird (vgl. 
mental training) und damit klinischen Interventionen prinzipiell zugänglich scheint [23, 27-29].

Mind-Body-Anwendungen stellen eine Möglichkeit dar, durch die Nutzung des Geistes (mind) bzw. mentaler und/oder potenzialorientierter Techniken physiologische Funktionen zu beeinflussen und die Gesundheit zu fördern [30]. Die MindBody-Medizin (MBM) sowie die Untersuchung Mind-Bodymedizinischer Fragestellungen und Anwendungen sind im angloamerikanischen Raum seit vielen Jahren etabliert [20, 31, 32]. Das amerikanische Gesundheitsministerium hat diesbezüglich eine formale Definition vorgenommen [30]. Auch im deutschsprachigen Raum kommen Interventionen unter diesem Begriff zunehmend zum Einsatz [33-38]. Grundsätzlich geht es bei der MBM um die Einbeziehung der Selbstregulation und Selbsthilfefähigkeit des Patienten in den Behandlungsverlauf, weswegen auch eine enge Beziehung zur klassischen Naturheilkunde bzw. zur integrativen (oder «komplementären») Medizin besteht [33, 34, 36, 39]. Dabei fokussiert die MBM nicht primär auf die «Krankenbehandlung» bzw. die Krankheit mit ihren pathoätiologischen Mechanismen, sondern vor allem auf die salutogenen bzw. gesundheitsförderlichen Anteile des Individuums (Selbst- und Autoregulation) sowie die gesundheitsbezogene Lebensqualität und das SWB $[32,37,39]$. Es besteht somit ein enger Bezug auch zu Konzepten der «Positiven Psychologie» und - theoretisch - zum Flourishing [40]. Unterschiedliche Studien konnten derweil positive Wirkungen der MBM auf chronische, stressbedingte und verhaltensbedingte Erkrankungen und auch auf Symptome bei Krebserkrankungen (vor allem psychologische Begleiterscheinungen) sowie Stimmung, Wohlbefinden und Lebensqualität zeigen [17, 18, 31, 35, 37, 38, 41-45]. Viele Erkrankungen sind stressassoziiert oder verhaltensbedingt [16, 39, 46, 47] - aber der Mensch ist in der Regel den Einflüssen und physiologischen Prozessen von Stress nicht völlig hilflos ausgeliefert. Menschen haben z.B. die Fähigkeit, den Einfluss von Stress zu reduzieren, Stress erfolgreich zu bewältigen und/oder die eigene Regulation (Selbst- bzw. Autoregulation) aktiv zu beeinflussen [16, 33, 47-49].

Aaron Antonovsky beschreibt in seiner Theorie der Salutogenese [50-52] (vgl. Kohärenzgefühl), dass nicht der Stressauslöser (Stressoren), sondern der individuelle Umgang mit dem Stress und die subjektive Bewältigung für Folgen und Ausmaß mitentscheidend sind. Verschiedene psychologische Copingstrategien, aber auch Persönlichkeitsmerkmale wie das Kohärenzgefühl, haben demnach einen Einfluss auf die Gesundheit, das emotionale SWB, die Lebensqualität und die Stressanfälligkeit $[52,53]$. Die gesundheitsbezogene Lebensqualität - psychometrisch messbar unter anderem über den SF-36- [54] und den SF-12-Fragebogen [55] - ist ein weiteres wichtiges Maß auch im Kontext vermuteter Wirkrichtungen des FlourishingKonstrukts. Ebenso kommen Aspekte der Achtsamkeit bzw. des Achtsamkeitserlebens hinzu, weswegen hier für die Überprüfung des Flourishing-Konstrukts jene Aspekte orientierend mit einbezogen wurden.
In unserer Studie haben wir den Einfluss von Flourishing, Achtsamkeit, Kohärenzgefühl und gesundheitsbezogener Lebensqualität bei Patienten einer Mind-Body-medizinischen Tagesklinik untersucht. Vertiefende Korrelationsanalysen zwischen den Faktoren generell sowie unter Einfluss der Intervention (multimodale MBM-Intervention und ihre Effekte anhand der weiteren Fragebögen) werden ausführlich und differenziert in Kürze in einer weiteren Publikation vorgestellt. In der vorliegenden Arbeit stehen folglich Einsatz und Machbarkeit sowie in der weiteren Betrachtung die Güte (Validität und Reliabilität) der deutschsprachigen Version des Flourishing-Fragebogens im Mittelpunkt.

Die Mind-Body-medizinische Tagesklinik bietet die Möglichkeit, die beschriebenen Zusammenhänge auf der Grundlage eines ressourcenorientierten salutogenen Ansatzes zu untersuchen. Die Tagesklinik umfasst ein standardisiertes multimodales Programm mit 12 Behandlungstagen. Das Gruppenangebot findet an einem Wochentag für $7 \mathrm{~h}$ über 3 Monate statt. Es werden Strategien zur Stressbewältigung und Verhaltensänderung (Bewegung, Ernährung, Entspannung, Meditation, Achtsamkeit, Kommunikation) und naturheilkundliche Selbsthilfestrategien vermittelt. Auf der Grundlage des genannten Ansatzes werden verschiedenste Erkrankungen wie rheumatische Erkrankungen, chronische Schmerzerkrankungen, funktionelle und chronische Magen-Darmerkrankungen, Stoffwechselerkrankungen, chronische Herz-Kreislauferkrankungen oder auch Erschöpfung behandelt. Für onkologisch erkrankte Patienten wurde eine separate Tagesklinik mit einem leicht veränderten Programm eingerichtet.

Das Ziel der vorliegende Studie ist a) die Überprüfung der Machbarkeit eines Einsatzes im medizinisch-klinischen Kontext (MBM) der im deutschsprachigen Raum erstmals zum Einsatz gebrachten deutschen Version (FS-D) des FlourishingFragebogens (FS) von Diener et al. [1] sowie b) die Analyse der Güte (Konstruktvalidität / inhaltliche Validität, konvergente und diskriminante Validität, Reliabilität) inklusive orientierender exploratorischer Faktorenanalyse.

\section{Methoden}

Studiendesign und Teilnehmer

Die Daten wurden mit einer Stichprobe von 43 Personen ohne Kontrollgruppe erhoben. Da der Therapieerfolg einer Mind-Body-medizinischen Tagesklinik (die Patienten sind in der Regel über einen Zeitraum von 12 Wochen an einem Tag der Woche in der Klinik) überprüft werden sollte, wurden den Probanden die gleichen Messinstrumente jeweils vor $\left(\mathrm{T}_{0}\right)$ und nach $\left(\mathrm{T}_{1}\right)$ der Intervention vorgelegt. Das Studienprotokoll wurde der Ethikkommission der Hochschule Coburg vorgelegt und von dieser genehmigt.

Der Fragebogenkomplex zur Datenerhebung setzte sich aus soziodemographischen Daten (6 Items), dem SF-12 (12 Items), der FS-D (8 Items), dem Freiburger Fragebogen zur Achtsamkeit (FFA; 14 Items) sowie der Sense of Coherence Scale 9 (SOC9; 9 Items) zusammen. 5 Tagesklinikgruppen nahmen an der Untersuchung teil. Die Befragungszeit richtete sich nach dem jeweiligen Beginn (August bis September 2011) und Ende (Oktober bis November 2011) der einzelnen Gruppen. Die Eig- 
nung der Patienten wurde durch ein strukturiertes Einzelinterview und ein medizinisches Anamnesegespräch vor Beginn des Aufenthalts in der Tagesklinik festgestellt. Die Teilnahme war für alle Patienten freiwillig.

Bei der Intervention, die dieser Untersuchung zugrunde lag, handelte es sich um einen Tagesklinikaufenthalt über 12 Wochen im ImmanuelKrankenhaus Berlin. Im Rahmen dieses standardisierten, multimodalen Ansatzes erhielten die zugewiesenen Patienten $(\mathrm{n}=48$; Drop-out $=5$; Teilnehmerverhältnis weiblich/männlich $=13: 1$; Mittelwert $(M)$ Alter: 52,3 Jahre) mit unterschiedlichen Diagnosen (28,9\% Erschöpfungssyndrom/Burnout, 21,1\% onkologische Erkrankung, 10,5\% Fibromyalgie, $4,7 \%$ chronisch-entzündliche Darmerkrankung und sonstige) Einweisungen und sammelten praktische Erfahrungen in der Mind-Body-Medizin (interventionelles Gruppensetting).

Die genauen Abläufe der Intervention werden in Kürze in einer anderen Publikation ausführlich dargestellt. Aus Gründen der Übersichtlichkeit und wissenschaftlichen Fokussierung entlang unserer Fragestellung wird hier auf eine genauere Beschreibung der Intervention und der beobachteten Effekte (Prä-post-Vergleich) verzichtet (siehe Ergebnisse).

\section{Untersuchungsinstrumente}

Die FS wurde von Ed Diener et al. [1] entwickelt. Die hier verwendete Version wurde zunächst von T.E., autorisiert von Ed Diener, ins Deutsche übersetzt (FS-D). Bei der Übersetzung wurde nach dem «4+2 Augen»-Prinzip vorgegangen. Dabei wurde von T.E. im Verlauf eine weitere Person (C.G.; Muttersprache Deutsch, Englisch als Zweitsprache) hinzugezogen und schließlich dem Originalautor eine Rückübersetzung vorgelegt. Diese wurde vom Originalautor autorisiert und die deutsche Fassung auf dessen Webseite angekündigt. Die FS besteht aus 8 Items. Diese beschreiben wichtige Aspekte der «Menschlichkeit» (siehe oben) wie positive Beziehungen, Kompetenzgefühl, Bedeutung und Sinn im Leben. Jedes Item wird mit einer 7-stufigen Skala von «Ich stimme stark/ voll zu» bis «Ich stimme absolut nicht zu» beantwortet. Alle Items sind als positive Aussage formuliert. Zur Beurteilung der Daten ist ein Summenscore zu bilden. Ein hoher Wert (maximal 56) bedeutet ein hohes Maß an Flourishing, ein niedriger Wert (mindestens 8 ) weist auf ein geringes $\mathrm{Ma}$ an Flourishing hin. Zur Einschätzung der konvergenten Validität bezogen Ed Diener und seine Arbeitsgruppe [1] eine Vielzahl an Messinstrumenten zum subjektiven Wohlbefinden, zu positiven und negativen Gefühlen, zum Optimismus, zu sozialen Beziehungen, zur Einschätzung von Kompetenz, zu Autonomie, Überlegenheit, Selbstwertgefühl sowie Sinn und Bedeutung im Leben ein. Die Analysen der Autoren zur Reliabilität der Skala ergaben gute bis zufriedenstellende Werte: Für die interne Konsistenz wurde ein Cronbachs Alpha $(\alpha)$ von 0,87 gemessen, die Untersuchung der Retest-Reliabilität ergab $r_{t t}=0,71$. Die geringe RetestReliabilität könnte eventuell mit der stark ausdifferenzierten Antwortskala (7 Stufen lassen sehr viel Variation im Antwortverhalten zu) und der nicht zufriedenstellenden Trennschärfe (mittlere Antwortkategorie) zusammenhängen. Dies müsste jedoch testtheoretisch weiter untersucht werden. Zur Ermittlung der konvergenten Validität wurden den Probanden der Normierungsstichprobe neben der FS die Ryff Scales of Psychological Well-Being [56] und Deci and Ryan's Basic Need Satisfaction (BNS) in General Scale [57] vorgelegt. Dabei korrelierte der FS mit den Subskalen des BNS zwischen $r_{t t}=0,54$ (autonomy) und $r_{t t}=0,67$ (competency). Der Zusammenhang des FS mit den Dimensionen der Ryff Scales lag zwischen $r_{t t}=0,43$ (autonomy) und $r_{t t}=0,73$ (mastery). Zur divergenten Validität lagen noch keine ausreichend belastbaren Daten vor. Die FS wurde mit einer Stichprobe von 689 Collegestudenten an 6 verschiedenen Orten über eine Monatsperiode evaluiert [1].

Die soziodemographischen Items setzten sich aus Geschlecht, Alter, Familienstand, Nationalität, höchster abgeschlossener Schulbildung und Erkrankung zusammen.

Um bei der Überprüfung des Therapieerfolgs der Tagesklinik inhaltlich valide Aussagen treffen zu können, wurden neben der FS-D weitere Fragebögen eingesetzt. Mit Hilfe dieser weiteren Skalen konnten zusätzlich Analysen zur konvergenten Validität der FS-D durchgeführt werden.
Der SF-12 ist die Kurzform des SF-36, der über die Medical Outcome Study (MOS) entwickelt wurde [54, 55, 58]. Der SF ist ein präzises und psychometrisch robustes Messinstrument zur Erfassung der gesundheitsbezogenen Lebensqualität $[54,55]$. In Studien zur integrativen Therapie bzw. zu multimodalen Therapieansätzen (in Komplementär- und MindBody-Medizin) hat sich insbesondere die gesundheitsbezogene Lebensqualität wiederholt als «kleinster gemeinsamer Nenner» und ausreichend veränderungssensitiver Parameter zur Beschreibung von Interventionseffekten erwiesen [37]. Das hängt unter anderem mit der Konstruktion des Fragebogens sowie dem expliziten Bezug zu Gesundheitsaspekten zusammen [57]. Allerdings gibt es derzeit noch keine allgemeingültige Definition der gesundheitsbezogenen Lebensqualität, was die klinische Interpretation der erhaltenen Ergebnisse erschwert - in jedem Fall bezieht sich die gesundheitsbezogene Lebensqualität auf das Individuum und dessen subjektive Wahrnehmung der Lebensqualität und hat so auch einen engen Bezug zum SWB $[57,58]$. Die Items des SF beziehen sich hauptsächlich auf die psychischen und physischen, weniger auf die sozialen Aspekte der Gesundheit [54]

Der FFA wurde von Harald Walach et al. entwickelt [24]. Die Forschungsgruppe konnte 2 Fragebogenversionen vorlegen, einen 30-ItemFragebogen und ein 14-Item-Fragebogen (Kurzform). Walach et al. konnten zeigen, dass der FFA prinzipiell für achtsamkeitserfahrene und -unerfahrene Teilnehmer geeignet ist. Allerdings haben sich in den letzten Jahren verschiedene Forschergruppen mit der Fragebogenanalytik zur Achtsamkeit und den grundsätzlich zu unterscheidenden Dimensionen intensiv beschäftigt und zum Teil neue Fragebögen und Kurzformen oder divergierende Übersetzungen vorgestellt. So hat man an der Universität Bern einen Fragebogen (Comprehensive Inventory of Mindfulness Experiences, CHIME) entwickelt, der in seiner Dimensionalität den Standard-Befragungsinstrumenten ähnelt, aber sprachlich besonders gut (robust) für alle Befragten - unabhängig von einer Meditationsvorerfahrung, d.h. diesbezüglich weniger anfällig - geeignet sein soll [59]. Ein anderer Aspekt der gegenwärtigen Diskussion ist, ob der FFA (in der englischen Version: FMI) bzw. der Five Facet Mindfulness Questionnaire (FFMQ) tatsächlich 4 bzw. 5 Facetten der Achtsamkeit unterscheidet oder ob es sich nicht allgemein (eindimensionale Achtsamkeit) um einen Generalfaktor bzw. eine Ein- oder maximal Zweidimensionalität handelt. Einige Forschergruppen bestätigen die 5-Faktor-Struktur [59, 60], andere eine 2-Faktor-Struktur, wobei hier divergierend entweder die beiden Bereiche Präsenz und Akzeptanz (FMI) [61] oder Aufmerksamkeit und Achtsamkeitserleben (Erfahrung; FFMQ) als zentrale der Aspekte der Achtsamkeit im Fragebogen unterschieden werden [62]. Die verringerte Dimensionalität bezieht sich dabei zumeist auf die abgeleiteten Kurzform-Versionen. Allen Instrumenten scheint jedoch gemein, dass die verschiedenen Facetten der Achtsamkeit sowohl einen Bezug zur Meditations-/Achtsamkeitserfahrung als auch zum subjektiven Wohlbefinden haben [63]. Die Freiburger Kurzform (14 Items) wird insbesondere für Bereiche empfohlen, in denen kein Wissen über den buddhistischen Hintergrund der Achtsamkeit zu erwarten ist [24, 64]. Dieser 14-Item-Fragebogen wurde daher in unserer Studie genutzt.

Die SOC wurde von Aaron Antonovsky entwickelt $[4,56]$. Neben der Vollversion mit 29 Items wurden mittlerweile 2 Kurzversionen mit 13 sowie 9 Items (unter anderem Leipzig Short Scale - SOC-L9) entwickelt und getestet [57, 58]. Dabei weisen die Validierungsergebnisse unter anderem zur 9-Item-Kurzversion auf einen Generalfaktor bzw. eine Eindimensionalität hin [67], die im Gegensatz zur Originalskala mit 3 Dimensionen steht, die sich allerdings in der prinzipiellen Komponenten-Faktorenanalyse (bei hoher interner Konsistenz: $\alpha=0,92$ ) als interkorreliert erwiesen hatten und insofern nicht eindeutig getrennt voneinander identifiziert werden konnten [68]. Untersuchungen aus Zürich zum arbeitsbezogenen Kohärenzgefühl (SOC) wiesen dagegen auf eine Zweidimensionalität hin. Insofern sind differenzierte Ableitungen, die sich eng am ursprünglichen 3-Faktoren-Modell orientieren, mit Vorsicht zu behandeln. In jedem Fall erfasst die SOC den Kohärenzsinn (Kohärenzgefühl) als eine generelle Einstellung im Leben (trait) und ist weniger situationsbe- 
Abb. 2. Lagemaße und Streuung zu $\mathrm{T}_{0}$ und $\mathrm{T}_{1}$ (KSK = körperliche Summenskala des SF-12; PSK = psychische Summenskala des SF-12; Flourishing $=$ deutsche Flourishing-Skala; FFA = Freiburger Fragebogen zur Achtsamkeit; SOC9 = Sense of Coherence Scale 9); y-Achse mit absoluten Summen- bzw. Subskalenwerten.

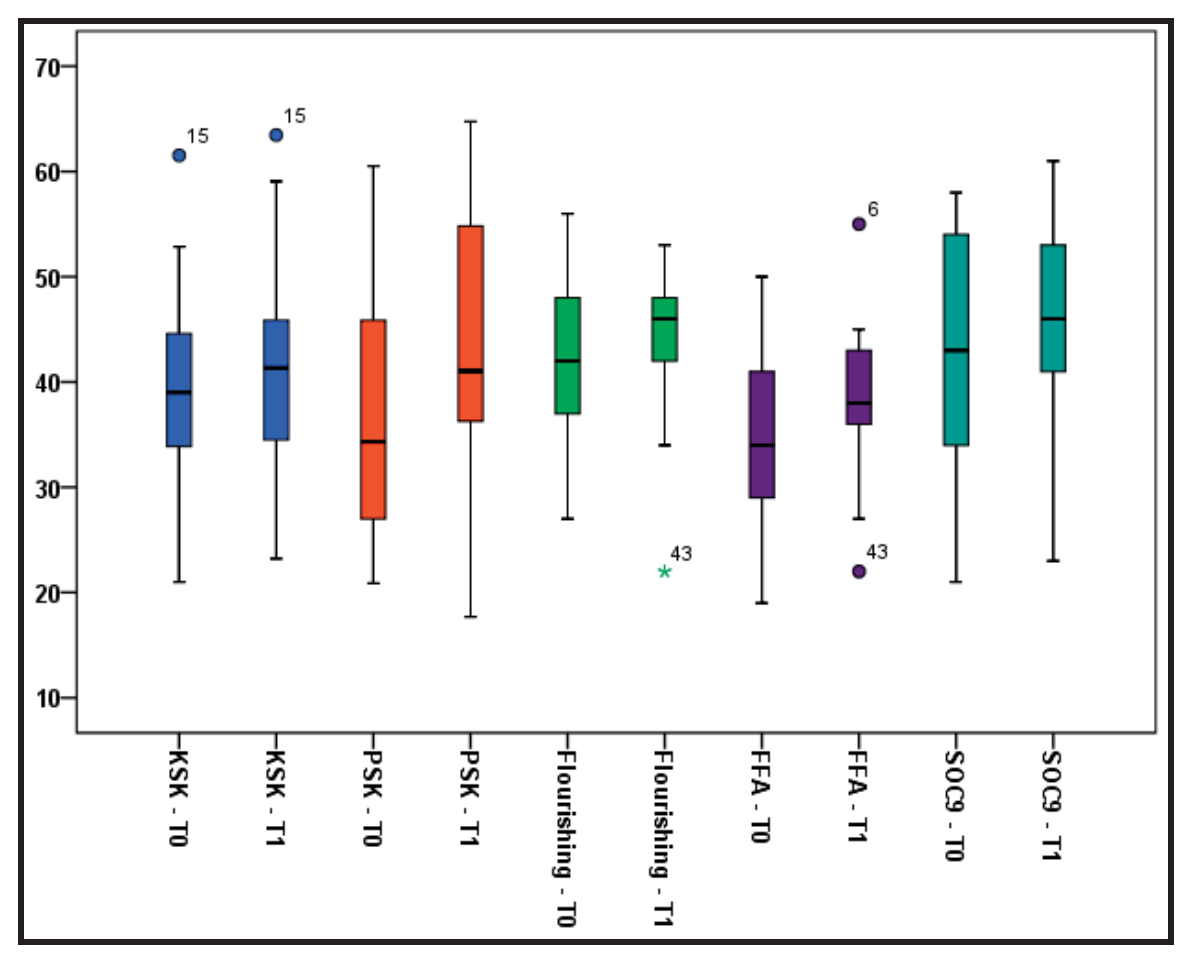

zogen (state) $[50-53,68]$. Die von uns verwendete Kurzfassung (SOC9) geht von einem Generalfaktor aus (siehe oben).

\section{Auswertung der Daten}

Die erhobenen Daten wurden mittels des Statistikprogramms SPSS 20.0 bearbeitet. Neben den Gesamtskalen wurden für den SF-12 2 Subskalen gebildet: körperlicher Gesundheitszustand (SF-12 K) und psychischer Gesundheitszustand (SF-12 P)

Da die Items der eingesetzten Untersuchungsinstrumente ausschließlich ordinales Skalenniveau aufweisen und die Stichprobe von N = 43 verhältnismäßig klein ist, wurde zur Reduktion von möglichen Messfehlern bei der Berechnung von Zusammenhängen statt einem parametrischen Verfahren (Pearson) der Rangkorrelationskoeffizient von Spearman (r) verwendet.

\section{Ergebnisse}

Die ausführliche Beschreibung der Studienpopulation sowie der deskriptiven Statistik erscheint in einer parallelen $\mathrm{Pu}$ blikation zu dieser Arbeit. Insgesamt nahmen 48 Patienten an unserer Studie teil. Im Verlauf der Intervention verließen $5 \mathrm{~Pa}$ tienten die Tagesklinik (Drop-out $=10,42 \%$ ). Folglich bestand die Stichprobe für die Datenanalyse aus 43 Teilnehmern.

\section{Lagemaße und Streuung}

Abbildung 2 zeigt die Verteilung zu beiden Messzeitpunkten anhand von Boxplots.

\section{Überprüfung des Therapieerfolgs}

Die Probanden erzielten nach der 12-wöchigen Intervention in der Tagesklinik bei allen eingesetzten Messinstrumenten signifikant bessere Resultate $(\mathrm{p} \leq 0,01)$ mit Ausnahme der SF $12 \mathrm{~K}$ (Tab. 1).
Tab. 1. Mittelwerte (M) zu $T_{0}$ und $T_{1}$, Standardabweichungen ( \pm SD), Signifikanzniveau (p-Wert)

\begin{tabular}{lrrrrl} 
& M-T $_{0}$ & \pm SD & M-T $_{1}$ & \pm SD & p-Wert \\
\hline SF-12 K & 38,60 & 10,68 & 40,79 & 10,28 & 0,118 \\
SF-12 P & 37,74 & 12,67 & 45,01 & 12,16 & 0,002 \\
FS-D & 40,28 & 8,18 & 43,18 & 6,75 & 0,006 \\
FFA & 34,54 & 7,70 & 38,08 & 6,34 & 0,010 \\
SOC 9 & 40,95 & 10,27 & 44,63 & 9,04 & 0,007 \\
\hline
\end{tabular}

SF-12 K = Subskala körperlicher Gesundheitszustand des SF-12; SF-12 P = Subskala psychischer Gesundheitszustand des SF-12; FS-D = deutsche Flourishing-Skala; FFA = Freiburger Fragebogen zur Achtsamkeit; SOC $9=$ Sense of Coherence Scale 9.

\section{Reliabilität (interne Konsistenz)}

Für die FS-D konnte zum Messzeitpunkt $\mathrm{T}_{0}$ ein $\alpha$ von 0,85 ermittelt werden, zu $\mathrm{T}_{1}$ lag die interne Konsistenz bei 0,79 . Damit konnte der hohe Wert der englischsprachigen Originalskala nicht ganz bestätigt werden. Die Ergebnisse liegen aber im akzeptablen bzw. guten Bereich. Eine Berechnung der Retest-Reliabilität erscheint nicht sinnvoll, da sich die Werte durch die Interventionsmaßnahmen der Klinik auf der FS-D verändern/verbessern sollten. Eine temporäre Stabilität kann konzeptionell nicht angenommen werden.

\section{Untersuchungen zur konvergenten bzw. diskriminanten Validität der FS-D}

Um Aufschluss darüber zu erhalten, inwiefern die FS-D mit den Konstrukten anderer Verfahren zusammenhängt bzw. sich von diesen unterscheidet, wurden für die einzelnen Skalen Zusammenhangsmaße ermittelt. Da die einzelnen Messinstru- 
Tab. 2. Korrelationen zwischen FS-D, SF-12 K, SF-12 P, FFA und SOC9 $\left(\mathrm{T}_{0}\right)$

\begin{tabular}{lllllll}
\hline & FS-D & SF-12 & SF-12 K & SF-12 P & FFA & SOC9 \\
\hline $\mathrm{r}$ & 1,000 & $0,565 * *$ & 0,188 & $0,434 *$ & $0,514^{* *}$ & $0,692 * *$ \\
Sig & - & 0,001 & 0,287 & 0,010 & 0,003 & 0,000 \\
$\mathrm{n}^{\mathrm{a}}$ & 40 & 34 & 34 & 34 & 31 & 39 \\
\hline
\end{tabular}

$\mathrm{r}=$ Rangkorrelationskoeffizient (Spearman Rho); Sig = Signifikanz; FS-D = deutsche FlourishingSkala; SF-12 = Kurzform des SF-36; SF-12 K = Subskala körperlicher Gesundheitszustand des SF-12; SF-12 P = Subskala psychischer Gesundheitszustand des SF-12; FFA = Freiburger Fragebogen zur Achtsamkeit; SOC9 = Sense of Coherence Scale 9 (Kurzform). **Die Korrelation ist auf dem 0,01-Niveau signifikant (zweiseitig).

*Die Korrelation ist auf dem 0,05-Niveau signifikant (zweiseitig).

${ }^{a}$ Wegen zum Teil nicht vollständig ausgefüllter Fragebögen konnten nicht alle Probanden in die Berechnung der Skalenwerte miteinbezogen werden. mente ausschließlich ordinalskalierte Variablen verwenden und angesichts der verhältnismäßig kleinen Stichprobe von $\mathrm{N}=43$ bei einem parametrischen Verfahren das offensichtliche Risiko von Messfehlern zu groß ist (inexakte Werte für Mittelwert (M) und Standardabweichung (SD)), wurde für die Berechnungen der Rangkorrelationskoeffizient von Spearman (r) verwendet. Dabei wurden nur die Daten zum Messzeitpunkt $\mathrm{T}_{0}$ in die Analysen einbezogen.

Die Korrelationen des FS-D mit den anderen Skalen (Tab. 2) liegen alle mit einer Ausnahme im mittleren Bereich (SF-12: $\mathrm{r}=0,57, \mathrm{p} \leq 0,01$; SF-12 P: $\mathrm{r}=0,43, \mathrm{p} \leq 0,05$; FFA: $\mathrm{r}=$ $0,51, \mathrm{p} \leq 0,01 ;$ SOC9: $\mathrm{r}=0,69, \mathrm{p} \leq 0,01)$. Lediglich mit der SF-12 $\mathrm{K}$ konnte kein signifikanter Zusammenhang ermittelt werden.

\section{Untersuchung der Faktorenstruktur der FS-D}

Zur vorläufigen Untersuchung der Dimensionalität der FS-D wurde eine exploratorische Faktorenanalyse (EFA; Hauptkomponentenanalyse, Eigenwerte $>1$; Rotationsmethode: Varimax) durchgeführt. Die Ergebnisse legen ein Zweifaktorenmodell nahe (Varianzaufklärung: 65,79\%). Die Resultate lassen jedoch aufgrund der kleinen Stichprobengröße nur erste Rückschlüsse auf eine Tendenz zu. Um robustere bzw. zuverlässigere Aussagen treffen zu können, ist es erforderlich, Faktorenanalysen mit Hilfe größerer Stichproben (z.B. doppelte Größe im Vergleich zur durchgeführten Studie) durchzuführen.

\section{Diskussion}

In unserer Studie wurde erstmals die deutsche Fassung FS-D der FS von Ed Diener et al. [1] eingesetzt. Die Anwendung der FS-D erwies sich innerhalb des Kollektivs von Tagesklinikpatienten (MBM) und der konkreten Fragestellung als prinzipiell durchführbar. Im Folgenden soll auf einige kritische Diskussionspunkte näher eingegangen werden.

Die erhobenen Daten beruhen auf subjektiven Einschätzungen der Teilnehmer. Hinzu kommt, dass verschiedene interne und externe Faktoren einen Einfluss auf die Beantwortung der Fragen haben können.
Die Stichprobe von 43 Teilnehmern ist relativ klein, aber für Interventionsstudien im vergleichbaren Umfeld nicht unüblich [18, 22, 23, 41, 42]. Für eine Validierungsstudie ist sie zur sicheren Berechnung einiger Parameter (weitergehende Itemanalysen wie Trennschärfe, Schwierigkeit, konfirmatorische Faktorenanalysen zur Bestimmung der Dimensionalität) nicht umfangreich genug; nicht zuletzt liegt auch ein Selektionsbias vor.

In der Evaluation des FS von Ed Diener et al. [1] wurden lediglich Studenten einbezogen. In unserer Erhebung erwies sich der FS-D für verschiedene Patientengruppen als geeignet (z.B. 29\% Erschöpfungssyndrom/Burnout, 21\% onkologische Erkrankung, 10\% Fibromyalgie). Allerdings können die Ergebnisse aufgrund der soziodemographischen Daten nur auf Frauen im Alter zwischen 40 und 75 Jahren bezogen werden (Publikation mit ausführlicher Beschreibung der Studienpopulation und Indikationen folgt in Kürze). Auch hier wäre es wünschenswert, bei weiteren Studien zur ergänzenden Itemanalytik der FS-D eine heterogenere und größere Stichprobe, d.h. einen eher experimentellen Rahmen zu wählen. Das war im vorliegenden Fall einer klinischen Machbarkeitsanalyse und Pilotvalidierung (im Tagesklinikkontext) nicht vorgesehen. Wir konnten allerdings dennoch Aussagen zur konvergenten und diskriminanten Validität, Reliabilität und zur exploratorischen Faktorenanalyse ermitteln.

Die Datenerhebung erfolgte über einen Zeitraum von durchschnittlich 12 Wochen. Die FS-D zeigte über diesen Zeitraum nachvollziehbare Veränderungen. Um die temporale Stabilität (Test-Retest-Reliabilität) der FS-D genauer zu bestimmen und von Interventionseffekten abzugrenzen, sind Folgestudien mit verschiedenen Messzeitpunkten und ohne zwischenzeitige Interventionen notwendig.

\section{Übersetzungsprozess}

Der von uns gewählte Übersetzungsprozess erfolgte auf Vorschlag des Originalautors der FS. Übersetzungen von neu eingeführten psychometrischen und sonstigen Befragungsinstrumenten zeigen jedoch immer wieder, selbst viele Jahre nach deren Einführung, dass Items auch missverstanden oder fehlinterpretiert werden können. Auch können sich kulturelle, genderbezogene, soziale oder altersabhängige Effekte erge- 
ben, die bei einer Primärübersetzung (oder auch bei der Originalpublikation) nicht erkennbar waren und/oder auf anderen und gegebenenfalls nicht immer zutreffenden/übertragbaren Vorannahmen beruhten (bzw. sich über die Zeit einstellen). Insofern ist es nicht unüblich, dass im Verlauf divergierende Versionen oder Übersetzungen (einzelner Items) sowie Alternativ- und Sonderversionen entwickelt und getestet werden. Dieses ist im Falle der FS auch zu erwarten. Allerdings müssen die einzelnen (zukünftigen) Versionen deutlich gekennzeichnet und ihrerseits validiert werden.

Unsere hier getestete und vorgestellte Version fungiert unter dem Akronym FS-D und hat in unserer Studie eine ausreichende Güte (und in Beziehung zur Originalpublikation nachvollziehbare Ergebnisse) gezeigt, was nach unserer Meinung ihren Einsatz für weitergehende Analysen und Messungen im deutschsprachigen Raum rechtfertigt.

\section{Lagemaße und Streuung}

Der Boxplot für Flourishing zeigt $z u T_{1}$ eine deutlich verdichtetere Verteilung als zu $\mathrm{T}_{0}$. Dies zeigt einen Gruppeneffekt für diesen Parameter. Die Verteilung $\mathrm{zu} \mathrm{T}_{0}$ ist weitestgehend symmetrisch. Allerdings zeigt sich ein verringertes Maximum. Dies kann auf den Erkenntniszuwachs der Teilnehmer durch den Aufenthalt in der Tagesklinik zurückzuführen sein, wodurch eventuell eine kritischere Beurteilung resultiert.

\section{Prä-post-Vergleich}

Der Prä-post-Vergleich des FS-D zeigt eine deutliche Steigerung von Flourishing nach einer Mind-Body-medizinischen Intervention (MBM-Tagesklinik). Conboy et al. [70] konnten eine Steigerung von Flourishing durch Yoga nachweisen, das zu den Mind-Body-medizinischen Anwendungen zählt. Es erscheint konzeptionell vorstellbar, dass Mind-Body-Anwendungen einen Einfluss auf die persönliche Entwicklung und das persönliche Wachstum (Selbsteinschätzung) haben können. Der Blick auf die Ressourcen einer Person könnte dabei helfen, «verborgene Potenziale» zu aktivieren (vgl. Flourishing-Konzept und Hintergründe zu Selbstregulation und Positiver Psychologie [40]).

\section{Konvergente und diskriminante Validität der FS-D}

FS-D und FFA weisen einen mittleren Zusammenhang auf. Catalino und Fredrickson [71] wiesen nach, dass Veränderungen der Achtsamkeit mit Veränderungen des Flourishing einhergehen. Dies passt mit den Ergebnissen von Conboy et al. zusammen [70]. Die Korrelation zwischen dem FS-D und dem SOC9 liegt ebenfalls im mittleren Bereich. Das Kohärenzgefühl ist eine konzeptionelle Voraussetzung für Gesundheit [50-52]. Flourishing beschreibt eine Art von Wohlbefinden $[1,7,12]$. Auf Grundlage dieser Aussagen kann man davon ausgehen, dass Kohärenz und Flourishing miteinander zusammenhängen könnten.

In unserer Analyse bestätigte sich der Zusammenhang zwischen Flourishing und Wohlbefinden durch die Korrelation von FS-D und SF-12 P.
Der fehlende Zusammenhang zwischen FS-D und SF-12 K erscheint plausibel, handelt es sich beim Flourishing doch um ein Konstrukt psychischer Befindlichkeit, das sich insbesondere auf mentale und kognitive Aspekte bezieht.

Damit zeigt sich, dass die FS-D auf der einen Seite mit wichtigen Sachverhalten, die im Zusammenhang mit Flourishing stehen, korreliert. Auf der anderen Seite weist sie Flourishing als eigenständiges Konstrukt nach, das sich nicht allein durch die Variablen Achtsamkeit, psychisches Wohlbefinden und Kohärenzgefühl erklären lässt. Somit lassen sich sowohl die konvergente als auch die diskriminante Validität der FS-D nachweisen.

Die Analysen zur internen Konsistenz der FS-D liegen im guten bzw. akzeptablen Bereich $\left(\mathrm{T}_{0}: \alpha=0,85 ; \mathrm{T}_{1}: \alpha=0,79\right)$. Damit konnte die Reliabilität der FS-D grundsätzlich bestätigt werden. Um robustere Aussagen machen zu können, müssten zur Evaluation der Skala jedoch größere Stichproben herangezogen werden.

Die Durchführung einer exploratorischen Faktorenanalyse lässt erste Hinweise auf eine zweidimensionale Struktur der FS-D erkennen. Zur weiteren Überprüfung des Zweifaktorenmodells sollten jedoch konfirmatorische Analysen an größeren Stichproben durchgeführt werden.

\section{Fazit}

Die FS-D zeigte sich innerhalb unserer Studie als ein valides und reliables Messinstrument für Flourishing bei Patienten verschiedenster Erkrankungen. Die Analyse zeigte nachvollziehbare Zusammenhänge mit Achtsamkeit, Kohärenz und psychischer Gesundheit. Generell existiert eine Vielzahl an Untersuchungsinstrumenten für Wohlbefinden. Die FS-D ist ein kurzer Fragebogen, der zur Erfassung von Flourishing oder eines ganzheitlichen Wohlbefindens genutzt werden kann. Je nach Fragestellung einer Untersuchung kann der FS-D Ergebnisse zur Entwicklung von Potenzialen und Ressourcen aufzeigen.

\section{Dank}

Diese Arbeit wurde unterstützt durch die Abteilung für Naturheilkunde am Immanuel-Krankenhaus Berlin-Wannsee und die Hochschule Coburg für angewandte Wissenschaften. Ganz besonders möchten sich die Autoren bei Prof. Dr. Ed Diener für die Überlassung der originalen FS und die Autorisierung für die deutschsprachige Version sowie Dr. Peter Malinowski (Liverpool) und Prof. Felicia Huppert (Cambridge) für die Unterstützung bedanken.

Ebenso gilt den Mitarbeitern der Tagesklinik, den teilnehmenden Patienten, Mitstudenten und Kollegen großer Dank.

\section{Disclosure Statement}

Die Autoren erklären hiermit, dass keine Interessenskonflikte im Zusammenhang mit der vorliegenden Arbeit vorliegen. 


\section{Literatur}

1 Diener E, Wirtz D, William T, Kim-Prieto C, Dongwon C, Oishi S, Biswas-Diener R: New well-being measures: short scales to assess flourishing and positive and negative feelings. Soc Indic Res 2010; 97:143-156.

2 Diener E, Diener M, Diener C: Factors predicting the subjective well-being of nations. J Pers Soc Psychol 1995;69:851-864.

$\checkmark 3$ Keyes CL:The mental health continuum: from languishing to flourishing in life. J Health Soc Behav 2002;43:207-222.

4 Diener E, Oishi S, Lucas RE: Personality, culture, and subjective well-being: emotional and cognitive evaluations of life. Annu Rev Psychol 2003;54:403425.

$\checkmark 5$ Diener E, Ng W, Harter J, Arora R: Wealth and happiness accross the world: material prosperity predicts life evaluation, whereas psychological prosperity predicts positive feeling. J Pers Soc Psychol 2010;99:52-61.

6 Keyes CLM, Haidt J: Flourishing: Positive Psychology and the Life Well-Lived. Washington, DC, American Psychological Association, 2003.

7 Fredrickson BL, Losada MF: Positive affect and the complex dynamics of human flourishing. Am Psychol 2005;60:678-686.

8 Seligman M: Authentic Happiness: Using the New Positive Psychology to Realize Your Potential for Lasting Fulfillment. New York, Free Press, 2002.

9 Seligman MEP: Flourish: A Visionary New Understanding of Happiness and Well-Being. New York, Atria, 2012.

10 Brown SL, Nesse RM, Vinokur AD, Smith DM: Providing support may be more beneficial than receiving it: results from a prospective study of mortality. Psychol Sci 2003;14:320-327.

11 Dunn E, Aknin LB, Norton MI: Spending money on others promotes happiness. Science $2008 ; 13$ 1687-1688.

12 Menk OL, Howerter A, Bell IR, Jackson N: Exploring measures of whole person wellness: integrative well-being and psychological flourishing. Explore (NY) 2010;6:364-370.

13 Diener E, Lucas RE, Scollon NC: Beyond the hedonic treadmill: revising the adaption theory of well-being. Am Psychol 2006;61:305-314.

14 Waterman AS: Two conceptions of happiness: contrasts of personal expressiveness (eudaimonia) and hedonic enjoyment. J Pers Soc Psych 1993;64: 678-691.

15 Malinowski P: Flourishing. Welches Glück hätten Sie gern? Positive Eigenschaften kultivieren und Schwierigkeiten meistern. München, Irisiana, 2010.

16 Esch T, Stefano GB: The neurobiology of stress management. Neuro Endocrinol Lett 2010;31:19-39.

17 Grossman P, Niemann L, Schmidt S, Walach H: Mindfulness-based stress reduction and health benefits. A meta-analysis. J Psychosom Res 2004; 57:35-43.

18 Schmith JE, Richardson J, Hoffman C, Pilkington $\mathrm{K}$ : Mindfulness-based stress reduction as supportive therapy in cancer care: systematic review. J Adv Nurs 2005;52:315-327.

19 Esch T, Fricchione GL, Stefano GB: The therapeutic use of the relaxation response in stress-related diseases. Med Sci Monit 2003;9:23-34.

20 Benson H: The relaxation response: therapeutic effect. Science 1997;278:1694-1695.

-21 Arias AJ, Steinberg K, Banga A, Trestman RL: Systematic review of the efficacy of meditation techniques as treatments for medical illness. J Altern Complement Med 2006;12:817-832.
22 Chang VY, Palesh O, Caldwell R, Glasgow N, Abramson M, Luskin F, Gill M, Burke A, Koopman $\mathrm{C}$ : The effects of a mindfulness-based stress reduction program on stress, mindfulness self-efficacy, and positive states of mind. Stress and Health 2006;20:141-147.

23 Ernst S, Esch SM, Esch T: The importance of mindfulness-based interventions in medical and psychotherapeutic care. Forsch Komplementmed 2009;16:296-303.

24 Walach H, Buchheld N, Buttenmüller V, Kleinknecht N, Schmidt S: Measuring mindfulness - the Freiburger Mindfulness Inventory (FMI). Pers Indiv Differ 2006;40:1543-1555.

25 Sauer S, Walach H, Kohls N: Gray's Behavioural Inhibition System as a mediator of mindfulness towards well-being. Pers Indiv Differ 2011;50:506-511.

26 Brown K, Ryan R: The benefits of being present: mindfulness and its role in psychological well-being. J Pers Soc Psychol 2003;84:822-848.

27 Ernst S, Welke J, Heintze C, Gabriel R, Zoellner A, Kiehne S, Schwantes U, Esch T: Effects of mindfulness-based stress reduction on quality of life in nursing home residents: a feasibility study. Forsch Komplementmed 2008;15:74-81.

28 Ludwig DS, Kabat-Zinn J: Mindfulness in medicine. JAMA 2008;300:1350-1252.

29 Sauer S, Lynch S, Walach H, Kohls N: Dialectics of mindfulness: implications for western medicine. Philos Ethics Humanit Med 2011; 6:10.

30 National Institutes of Health. Definition: mind-bodymedicine. http://nccam.nih.gov/health/whatiscam/ (Zugriff am 19.07.2013).

31 Astin JA, Shapiro SL, Eisenberg DM, Forys KL: mind-body medicine: state of the science, implication for practice. J Am Board Fam Pract 2003;16: 131-147.

32 Komaroff AL (ed): Mind/Body Medicine: A Special Health Report. Boston, Harvard Health Publications, 2001.

33 Dobos G, Paul A (eds): Mind-Body-Medizin. Die moderne Ordnungstherapie in Theorie und Praxis. München, Elsevier, 2011.

34 Dobos G, Altner N, Lange S, Musial F, Langhorst J, Michalsen A, Paul A: Mind-Body-Medicine als Bestandteil der Integrativen Medizin. Bundesgesundheitsbl - Gesundheitsforsch - Gesundheitsschutz 2006;49:723-728

35 Esch T, Sonntag U, Esch SM, Thees S: Stress management and mind-body medicine: a randomized controlled longitudinal evaluation of students' health and effects of a behavioral group intervention at a middle-size german university (SMMESH). Forsch Komplementmed 2013;20:129-137.

36 Esch T, Esch SM: Stressbewältigung mithilfe der Mind-Body-Medizin. Trainingsmanual zur Integrativen Gesundheitsförderung. Berlin, Medizinisch Wissenschaftliche Verlagsgesellschaft, 2013.

37 Ostermann T, Langhorst J, Beer AM: The effects of integrative in-patient treatments on patients' quality of life: a meta-analysis. Evid Based Complement and Alternat Med 2013;2013:416510.

-38 Spahn G, Choi KE, Kennemann C, Lüdtke R, Franken U, Langhorst J, Paul A, Dobos GJ: Can a multimodal mind-body program enhance the treatment effects of physical activity in breast cancer survivors with chronic tumor-associated fatigue? A randomized controlled trial. Integr Cancer Ther 2013;12:291-300.

39 Esch T: Mind-Body-Medizin: Stress, Stressmanagement und Gesundheitsförderung. Komplement Integr Med 2008;1:35-39.
40 Esch T: Die Neurobiologie des Glücks. Wie die Positive Psychologie die Medizin verändert. Stuttgart, Thieme, 2012.

41 Schure MB, Christopher J, Christopher S: Mindbody medicine and the art of self-care: teaching mindfulness to counseling students through yoga, meditation, and qigong. J Couns Dev 2008;86:47-56.

42 Esch T, Duckstein J, Welke J, Stefano GB, Braun V: Mind/body techniques for physiological and psychological stress reduction: stress management via tai chi training - a pilot study. Med Sci Monit 2007;13:488-497.

43 Elsenbruch S, Langhorst J, Popkirowa K, et al.: Effects of mind-body therapy on quality of life and neuroendocrine and cellular immune functions in patients with ulcerative colitis. Psychother Psychosom 2005;74:277-287.

44 Hadhazy VA, Ezzo J, Creamer P, Berman BM: Mind-body therapies for the treatment of fibromyalgia. A systematic review. J Rheumatol 2000;27: 11-18.

45 Hoffmann B, Moebus S, Michalsen A, Paul A, Spahn G, Dobos GJ, Jöckel KH: Health-related control belief and quality of life in chronically ill patients after a behavioral intervention in an integrative medicine clinic - an observational study. Forsch Komplementmed 2004;11:159-170.

46 Esch T: Stress, adaptation, and self-organization: balancing processes facilitate health and survival. Forsch Komplementarmed Klass Naturheilk 2003; 10:330-341.

47 Esch T: Health in stress: change in the stress concept and its significance for prevention, health and life style. Gesundheitswesen 2002;64:73-81.

48 Benson H, Stuart EM: The Wellness Book. The Comprehensive Guide to Maintaining Health and Treating Stress-Related-Illness. New York, Fireside, 1993.

49 Kaluza G: Stressbewältigung. Trainingsmanual zur psychologischen Gesundheitsförderung. Heidelberg, Springer Medizin, 2009.

50 Antonovsky A, Franke A: Salutogenese. Zur Entmystifizierung der Gesundheit. Tübingen, DGVT, 1997.

51 Richardson CG, Ratner PA: Sense of coherence as a moderator of the effects of stressful life events on health. J Epidemiol Community Health 2005; 59:979-984.

52 Eriksson M, Lindström B: Antonovsky's sense of coherence scale and its relation with quality of life: a systematic review. J Epidemiol Community Health 2007;61:938-944.

53 Nahlén C, Saboonchi F: Coping, sense of coherence and the dimension of affect in patients with chronic heart failure. Eur J Cardiovasc Nurs 2010; 9:118-125.

54 Bullinger M, Kirchberger I: SF-36 Fragebogen zum Gesundheitszustand. Handanweisungen. Göttingen, Hogrefe, 1998.

55 Müller-Nordhorn J, Roll S, Willich SN: Comparison of the short form (SF)-12 health status instrument with the SF-36 in patients with coronary heart disease. Heart 2004;90:523-527.

56 Ryan RM, Deci EL: Self-determination theory and the facilitation of intrinsic motivation, social development, and well-being. Am Psychol 2000;55: 68-78.

57 Bitzer EM: Die Perspektive der Patienten-Lebensqualität und Patientenzufriedenheit; in Schwartz FW, Walter U, Siegrist J, Kolip P, Leidl R, Dierks M-L, Busse R, Schneider N: Public Health - Gesundheit und Gesundheitswesen. München, Urban und Fischer, 2003. 
58 Kurth BM: Gesundheitsbezogene Lebensqualität; in Bundeszentrale für gesundheitliche Aufklärung (ed): Leitbegriffe der Gesundheitsförderung. Glossar zu Konzepten, Strategien und Methoden in der Gesundheitsförderung. Schwabenheim a.d Selz, Peter Sabo, 2003.

59 de Bruin EI, Topper M, Muskens JG, Bögels SM Kamphuis JH: Psychometric properties of the Five Facets Mindfulness Questionnaire (FFMQ) in a meditating and non-meditating sample. Assessment 2012;19:187-197.

60 Bohlmeijer E, ten Klooster PM, Fledderus M, Veehof M, Baer R: Psychometric properties of the five facet mindfulness questionnaire in depressed adults and development of a short form. Assessment 2011;18:308-320.

61 Büssing A, Walach H, Kohls N, Zimmermann F, Trousselard M: Conscious presence and self control as a measure of situational awareness in soldiers - a validation study. Int J Ment Health Syst 2013;7:1.

62 Tran US, Glück TM, Nader IW: Investigating the Five Facet Mindfulness Questionnaire (FFMQ) construction of a short form and evidence of a two-factor higher order structure of mindfulness. J Clin Psychol 2013;DOI:10.1002/jclp.21996.
63 Baer RA, Smith GT, Lykins E, Button D, Krietemeyer J, Sauer S, Walsh E, Duggan D, Williams JM: Construct validity of the five facet mindfulness questionnaire in meditating and nonmeditating samples. Assessment 2008;15:329-342.

64 Walach H, Buchheld N, Buttenmüller V, Kleinknecht N, Grossmann P, Schmidt S: Empirische Erfassung der Achtsamkeit - die Konstruktion des Freiburger Fragebogens zur Achtsamkeit (FFA) und weitere Validierungsstudien; in Heidenreich T, Michalak J (eds): Achtsamkeit und Akzeptanz in der Psychotherapie. Ein Handbuch. Tübingen, dgvt, 2004.

65 Antonovsky A:The structure and properties of the sense of coherence scale. Soc Sci Med 1993;36:725733.

66 Flannery RB Jr, Perry JC, Penk WE, Flannery GJ: Validating Antonovsky's Sense of Coherence Scale. J Clin Psychol 1994;50:575-577.

67 Schumacher J, Wilz G, Gunzelmann T, Brähler E: Die Sense of Coherence Scale von Antonovsky. Teststatistische Überprüfung in einer repräsentativen Bevölkerungsstichprobe und Konstruktion einer Kurzskala. Psychother Psychosom Med Psychol 2000;50:472-482.
68 Schnyder U, Büchi S, Sensky T, Klaghofer R: Antonovsky's sense of coherence: trait or state? Psychother Psychosom 2000;69:296-302.

69 Bauer GF, Jenny GJ: Development, implementation and dissemination of occupational health management (OHM): putting salutogenesis into practice; in McIntyre S, Houdmondt J (eds): Occupational Health Psychology. European Perspectives on Research, Education and Practice, vol 2. Castelo da Maia, European Academy of Occupational Health Psychology (EA-OHP), 2007, pp 219-250.

70 Conboy LA, Wilson A, Braun T: Moving beyond health to flourishing: the effects of yoga teacher training. TheScientificWorldJournal 2012;10:788795.

71 Catalino LI, Fredrickson BL: A Tuesday in the life of a flourisher: the role of positive emotional reactivity in optimal mental health. Emotion 2011;11 938-950. 